\title{
Recepción de la ficción televisiva norteamericana en España. El caso de Friends
}

\author{
Mar Grandío ${ }^{1}$
}

\begin{abstract}
Resumen: Enmarcado dentro de los Estudios de Recepción, esta investigación estudia la audiencia de Friends en España entorno al concepto de entretenimiento y la actitud del espectador hacia el universo "americano representado". En primer lugar, este artículo proporciona una descripción general de la audiencia española de Friends, sobre todo centrada en su perfil sociodemográfico y hábitos de consumo. En segundo lugar, se detallará la relación que se establece entre la audiencia y el universo "americano representado". En esta investigación se combinan métodos cuantitativos y cualitativos con el empleo de 2.494 encuestas online, dos grupos de discusión y diez entrevistas en profundidad.
\end{abstract}

Palabras claves: Estudios de Recepción, ficción televisiva, Friends.

Resumo: Situada dentro dos Estudos de Recepção, esta pesquisa investiga a audiência de Friends na Espanha, focalizando o conceito de entretenimento e a atitude do espectador em relação ao universo "americano representado". Em primeiro lugar, este artigo oferece uma descrição geral da audiência espanhola de Friends, centrada, sobretudo, em seu perfil sociodemográfico e seus hábitos de consumo. Em segundo lugar, será detalhada a relação que se estabelece entre a audiência e o universo "americano representado". Nesta investigação, são combinados métodos quantitativos e qualitativos com o emprego de 2.494 entrevistas online, dois grupos de discussão e dez entrevistas em profundidade.

Palavras-chave: Estudos de Recepção, ficção televisiva, Friends.

\begin{abstract}
Within the reception studies, this article is about the viewers of the situation comedy Friends in Spain. Taking into account a situation comedy, pleasure is regarded as a light viewing to laugh and forget about the problems. First of all, I will look into consume of this TV show. Why does the Spanish audience want to watch this comedy? Which kind of gratification do they get? Secondly, Spanish viewer's attitude towards this "American universe" will be described. This research uses a combination of quantitative and qualitative methods to study the distinctive viewing of the Spanish fans of this TV sitcom. In the first phase, existing data comes from 2.494 online questionnaires. This analysis provides a context for the qualitative data that is based on two focus groups and ten in-depth interviews.
\end{abstract}

Key words: Reception Studies, American television fiction, Friends.

\footnotetext{
${ }^{1}$ Profesora doctora de la Universidad Católica San Antonio de Murcia, en España. E-mail: mgrandio@pdi.ucam.edu.
} 


\section{Introducción. Ficción televisiva norteamericana y Estudios de Recepción: entretenimiento y "americanización”}

La ficción norteamericana en España ha vivido en los últimos quince años un auténtico “amanecer”. La audiencia española prefiere las series nacionales y el prime time español ha estado tradicionalmente lleno de ficción doméstica dirigida principalmente a toda la familia. Sin embargo, series como Expediente X (Fox, 19932002), Twin Peaks (ABC, 1990-1991) o Ally MacBeal (FOX, 1997-2002) iniciaron en la década de los 90 una senda de logros en nuestro país que se ha coronado en la actualidad con grades éxitos como CSI (CBS, 2000-), House (FOX, 2004-) o Anatomía de Grey (ABC, 2005-), programas que llegan a ser, incluso, seña de identidad para las cadenas que las emiten en España. En este sentido, tal vez una de las series norteamericanas con más éxito en nuestro país en la última década haya sido la comedia de situación Friends (NBC, 1994-). Esta sitcom empezó a emitirse en Estados Unidos el 14 de septiembre de 1994 y narra la vida de seis veinteañeros neoyorkinos. En España, aún emitiéndose por la cadena de pago Canal + en España y posteriormente en reposiciones en la cadena generalista Cuatro, ha conseguido ser un auténtico fenómeno entre los jóvenes españoles desde su estreno en 1997. Es por ello que una vez finalizadas sus diez temporadas en 2005 en España, se planteó la idoneidad de un estudio de recepción sobre esta serie en nuestro país. La relevancia de una investigación de estas características era doble. Por un lado, se conocería cómo una serie de éxito mundial era recibida e interpretada por una audiencia local y, por otro lado, se aportarían datos sobre la recepción televisiva de la ficción norteamericana en España, un terreno prácticamente inexplorado.

Si miramos el panorama académico internacional dentro de los denominados Estudios de Recepción, especialmente el ámbito anglosajón, observamos cómo son cuantiosos los trabajos referentes a la ficción televisiva convirtiéndose, incluso, en una de las áreas más investigadas. En concreto, la telenovela ha sido uno de los géneros estrellas objeto de estudio (Ang, 1985; Schrøder, 1988; Liebes, Katz, 1990; Baym 2000; Spence, 2005), aunque también existen trabajos relacionados con otros géneros televisivos como las comedias o el drama (Biltereyst, 1991; Jhally, Lewis, 1992; McKienley, 1997; Brook, 2004, Kuipers, 2006; Dhoest, 2007). En el caso español, 
apenas existen trabajos sobre la recepción de las series de ficción (Grandío, 2007) y los más característicos se centran en la construcción de la identidad individual adolescente desde una perspectiva psicológica (Huertas y França, 2001; Figueras, 2006; Montero, 2006; Pindado, 2006), más que un estudio sobre la repercusión social y cultural de dicha recepción.

Uno de los temas más tratados en las investigaciones sobre la recepción de ficción televisiva es el entretenimiento o disfrute. De hecho, es sencillo entender que la gente sigue un determino programa televisivo para pasar un buen rato y divertirse. El concepto de pleasure aparecía específicamente en la década de los 80 dentro de los Estudios de Recepción. Al intentar explicar cómo se enfrentaban los públicos a determinados productos audiovisuales, se utilizó el concepto de entretenimiento para conceptualizar y explicar el placer o disfrute televisivo. Concretamente, la primera literatura de este campo intentaba dar respuesta a por qué la audiencia femenina seguía habitualmente los seriales románticos en televisión. Curiosamente, la obra que incorpora el tema del pleasure al debate de los Estudios de Recepción proviene del ámbito de la literatura. Reading the Romance. Women, Patriarchy, and Popular Literature de Janice Radway aplica el concepto del pleasure a las ficciones literarias en un afán por explicar por qué las mujeres disfrutaban leyendo novelas románticas (1984). Sin embargo, la experiencia del disfrute televisivo permanece aún difusa en el ámbito académico. Casi un cuarto de siglo después, el debate sobre qué es realmente el entretenimiento televisivo y qué significa para su público sigue estando abierto, algo que deja entrever la importancia y complejidad de este objeto de estudio.

Por otro lado, una de las cuestiones más analizadas respecto a la ficción norteamericana en el extranjero ha sido su impacto social. Se han tratado, sobre todo, cuestiones relacionadas con conceptos como el imperialismo cultural y su impacto sobre las identidades culturales de las naciones. En concreto, la gran cantidad de ficción norteamericana en las parrillas mundiales hace investigar a algunos académicos sobre el proceso de americanización o homogenización de las culturas nativas (Katz and Liebes, 1990; Biltereyst, 1991; Harrington, Bielby, 2005). Sin embargo, hay investigadores que consideran que las audiencias interpretan las series globales-por lo sobre todo norteamericanas- con el particular prisma de su propia cultura nacional (McQuail, 1997; Olson, 1999, Chitnis, 2006). 
En esta línea, este artículo pretende aportar algo de luz en este terreno con el análisis concreto de la recepción de la comedia de situación Friends en España y se prestará especial atención a dos cuestiones: en primer lugar, al concepto de entretenimiento y a la actitud de los espectadores hacia los valores representados en Friends.

\section{Estudio de recepción de Friends en España}

La investigación de la audiencia de esta comedia de situación en España ha seguido tres etapas. La primera corresponde a una encuesta que estuvo accesible a través de Internet durante seis meses y que se distribuyó entre los fans de la serie. Este cuestionario estuvo disponible desde febrero de 2005 aunque, a efectos de recopilación de datos, se cerró el 10 de abril cuando se habían recibido 2.494 encuestas. La segunda fueron dos grupos de discusión con varios seguidores de la serie. En la tercera y última se realizaron diez entrevistas en profundidad con las que se indagó en el concepto de entretenimiento. En este artículo se combinan métodos cuantitativos y cualitativos; además, se utilizará un modelo basado en los usos y gratificaciones de los seguidores así como los valores culturales implicados en el proceso de recepción.

Aunque este estudio no pretendía en sus orígenes ser un estudio exhaustivo del fenómeno fan de esta serie en España, la recogida de datos nos llevó a centrarnos en un público entusiasta de la serie. En este apartado, basado en exclusiva en las encuestas online, se intentará ofrecer una aproximación a las características de este grupo específico de audiencia. En primer lugar, se dará una descripción general de los fans de Friends en España. Después, se pondrá de manifiesto cómo es un público bastante homogéneo caracterizado principalmente por un gran consumo de la serie y una alta gratificación.

\subsection{Público homogéneo caracterizado por un gran consumo de la serie y una alta gratificación}

En este apartado se hará alusión a los aspectos sociodemográficos de los seguidores de la serie para luego tratar de las cuestiones relacionadas con el consumo, 
grado de gratificación, personaje favorito e historias representadas en serie para la audiencia.

La difusión de la encuesta por la Red ha conseguido que se obtuviera un perfil sociodemográfico amplio y representativo de los seguidores de Friends en España. En este sentido, se puede afirmar que Friends en España ha sido un fenómeno juvenil y sin diferencias reseñables en función del sexo. Por un lado, el 60,3\% de los encuestados tienen entre 16 y 25 años y la horquilla delimitada entre los 25 y 35 años agrupa a $33,2 \%$ de los espectadores encuestados. Por sexo, el 60,9\% son hombres frente al 39,1\% de mujeres. Si tenemos en cuenta la variable de los estudios realizados, el 63,9\% ha hecho algún tipo de estudios universitarios, el 19,8\% posee el título de enseñanza secundaria y el 14,6\% ha realizado cursos de formación profesional.

En relación al seguimiento, un 84,7\% por ciento de las personas encuestadas definió su grado de seguimiento como alto, es decir, que había visto prácticamente toda la serie. Solamente el $10,8 \%$ considera que su consumo ha sido medio ya que ha visto algunas temporadas pero no la serie completa. El 4,5\% había visto la serie ocasionalmente. [Véase tabla 1].

Tabla 1: Grado de seguimiento de la serie

\begin{tabular}{|c|c|}
\hline SEGUIMIENTO & PORCENTAJE \\
\hline Alto & $84,7 \%$ \\
\hline Medio & $10,8 \%$ \\
\hline Bajo & $4,5 \%$ \\
\hline Total & $100 \%$ \\
\hline
\end{tabular}

(Fuente: Elaboración propia)

Para valorar el consumo, interesaba sobre todo medir el grado de visionado por DVDs de la serie frente al consumo tradicional por televisión. Debido a la estrategia de programación de Canal Plus, la emisora de pago por la cual se emitió esta serie, es significativo del fenómeno Friends en España el consumo por DVDs o episodio 
grabados $^{2}$. Para cuantificar esta tendencia, se les preguntó directamente si habían visto Friends por DVD. El 29,8\% contestó que había visto todas las temporadas comercializadas, el 30,6\% algunas temporadas y el 39,6\% declaraba haberlo visto solamente por televisión. [Tabla 2]. Por lo tanto, más del $60 \%$ de los encuestados ha visto todas o algunas de las temporadas comercializadas en DVD frente al casi $40 \%$ que exclusivamente lo había visto por televisión. Otra variable interesante respecto al consumo era conocer si había primado un visionado colectivo o individual de Friends. El 43,8\% de los encuestados declara que ha visto la serie habitualmente solo, el 23,2\% afirma que la ha visto con amigos y el 33\% en familia.

Tabla 2: Consumo de la serie por DVDs

\begin{tabular}{|c|c|}
\hline CONSUMO & PORCENTAJE \\
\hline Todas las temporadas por DVD & $29,8 \%$ \\
\hline Algunas temporadas por DVD & $30,6 \%$ \\
\hline Sólo por televisión & $39,6 \%$ \\
\hline Total & $100 \%$ \\
\hline
\end{tabular}

(Fuente: Elaboración propia)

Los encuestados también se caracterizan por una alta gratificación en términos de entretenimiento y humor. El 97,9\% considera que Friends es una serie tremendamente entretenida o muy entretenida frente únicamente el $2 \%$ que dice que es entretenida sin más. [Tabla 3].

\footnotetext{
${ }^{2}$ Es precioso señalar aquí que la serie empezó a emitirse en 1997 en España por el canal de pago Canal Plus en abierto a las 15:00 horas. Ha sido repuesta en multitud de ocasiones en este horario. Los capítulos nuevos los emitían en codificado para gente abonada a las 20:00 horas los domingos.
} 
Tabla 3: Grado de entretenimiento de la serie

\begin{tabular}{|c|c|}
\hline ENTRETENIMIENTO & PORCENTAJE \\
\hline Tremendamente entretenida & $82,8 \%$ \\
\hline Muy entretenida & $15,1 \%$ \\
\hline Entretenida sin más & $2 \%$ \\
\hline Poco entretenida & $0,2 \%$ \\
\hline Nada entretenida & $0 \%$ \\
\hline Total & $100 \%$ \\
\hline
\end{tabular}

(Fuente: Elaboración propia)

En términos humorísticos, el 97,5\% responde que le parece además tremendamente graciosa o muy graciosa frente al 2,3\% que afirma que es algo graciosa. [Véase Tabla 4].

Tabla 4: Grado de humor de la serie

\begin{tabular}{|c|c|}
\hline HUMOR & PORCENTAJE \\
\hline Tremendamente graciosa & $61,5 \%$ \\
\hline Muy graciosa & $36 \%$ \\
\hline Graciosa sin más & $2,3 \%$ \\
\hline Poco graciosa & $0,2 \%$ \\
\hline Nada graciosa & $0 \%$ \\
\hline Total & $100 \%$ \\
\hline
\end{tabular}

(Fuente: Elaboración propia)

Respecto al contenido, en otra pregunta se les dio una serie de características para que indicaran qué era lo que más valoraban de esta comedia de situación. Aquí, un 33,5\% señaló que lo que más valoraba eran las situaciones cómicas creadas, un 31\% los diálogos y un 22,3\% las relaciones que se establecían entre los personajes. También interesaba conocer qué tipo de historias se contaban en Friends. Por ello, se pidió a los 
encuestados que las definiesen. Se les dio a elegir entre una serie de conceptos que bien podrían captar la esencia de la narración. Ante esta cuestión, el 63\% piensa que, ante todo, las historias narradas en esta comedia de situación son universales, el 17,3\% considera las tramas propias de la ficción frente al 6,5\% que afirma que las historias representadas en Friends son exclusivamente americanas [Véase tabla 5]. Asimismo se les preguntó, específicamente, cuál era su personaje favorito y por qué lo elegían como tal. Al respecto, hay que señalar que los tres personajes masculinos aparecen colocados en los primeros puestos mientras que los tres personajes femeninos se sitúan al final. El 33,5\% de los encuestados eligió a Chandler como personaje favorito, el 26,4\% señalaron a Joey y un 12,8\% a Ross. Rachel acapara un 11,8\%, Phoebe un 10,5\% y Mónica un 4,7\%.

Tabla 5: Tipo de historia

\begin{tabular}{|c|c|}
\hline TIPO DE HISTORIA & PORCENTAJE \\
\hline Exclusivamente americana & $6,5 \%$ \\
\hline Temas universales & $63 \%$ \\
\hline Propias de la ficción & $17,3 \%$ \\
\hline Otros & $13,1 \%$ \\
\hline Total & $100 \%$ \\
\hline
\end{tabular}

(Fuente: Elaboración propia)

\subsection{Necesidad de entretenimiento, consumo rápido y gratificación inmediata}

La audiencia ha visto Friends por un entretenimiento fundamentado en el humor. Aunque la mayoría de las veces los seguidores veían la serie de una manera casual, en muchos casos se convirtió en una auténtica actividad pensada al consumir la serie por mero entretenimiento. Es decir, los espectadores buscaban diversión, sobre todo reírse incluso “a carcajadas”:

A mí lo que me gusta de Friends es que no hay un solo capítulo en el que, ya no te digo reír, ¡no hay un solo capítulo en el que no sueltes una carcajada que te quedes 
a gusto! Yo creo que con todos los capítulos, da igual cual cojas, te ríes un montón. Te ríes con todos.

(Grupo de discusión, 19 años, hombre, estudiante universitario)

En esta línea, son muchos los espectadores que siguen esta tendencia de resaltar de Friends que les hace “reírse a carcajadas”. Merece la pena subrayar cómo este último espectador explica que se queda “a gusto” cuando se ríe. La búsqueda de humor se satisface a través de la risa. Cabe pensar que reírse de esta manera tan abierta les proporciona una sensación física de bienestar. Por lo tanto, se podría hablar de un primer nivel con una gratificación física por lo que de liberación de tensión corporal conlleva la risa. Como consecuencia de la risa se obtiene la relajación. De hecho, bastantes seguidores comentan que ver Friends era una actividad relajante. Con este planteamiento de partida tan centrado en la gratificación, es entendible cómo algunos espectadores afirman que Friends les decepciona cuando no les hace reír:

Cuando se ha intentado meter alguna trama un poco más profunda, como puede ser cuando Chandler y Mónica quieren adoptar un hijo, yo creo que ahí Friends se ha desvirtuado. No es que no me interese ese tema. Es que rompe con la tónica de la serie. Yo busco la risa inmediata. Reunirme con mis amigos y echarme unas risas. Por eso creo que no procede que se pongan de repente con problemas existenciales de mayor o menor grado. Es que en Friends buscas reírte. Y si no me río, me quedo mal. Me quedo decepcionada.

(Entrevista en profundidad, 26 años, mujer, productora)

Esta espectadora pone de manifiesto otra de las características que se ha venido comentando: la inmediatez. A la audiencia le gusta la agilidad y rapidez de este producto audiovisual de escasa media hora. Por lo tanto, las mismas características del producto hacen que su consumo sea también rápido y dinámico. No en vano, la brevedad del formato y la intensidad de la experiencia audiovisual han generado que los espectadores tengan casi la necesidad de ver varios episodios seguidos. Por lo tanto, si el consumo de este producto es rápido, se puede ya afirmar que la gratificación es inmediata. Algún espectador compara la esta serie con una píldora y dice que, “Friends es una píldora pequeña pero efectiva. Media horita de buen humor sin complicaciones”. En definitiva, se podría hablar de una búsqueda de humor, consumo rápido y una gratificación física instantánea en forma de risa y relajación. 
Sin embargo, el humor de esta serie también ofrece un abanico de gratificaciones que van más allá de la puramente física. De hecho, existe un nivel más profundo que la gratificación producida por la risa y que se encuentra en un ámbito más incorporal. Aparte de la motivación evidente de los espectadores para acercase a esta serie para reírse, los espectadores hablan de ver Friends por la atracción hacia un mundo que muestra la parte simpática y amable de la realidad, tal y como señala este espectador:

Fue una serie que me enganchó por el planteamiento de fondo que tenía. Sobre todo, por esa visión simpática de la realidad. El día a día normal de una serie de personas muy diferentes que confluyen por amistad. Yo creo que esta visión amable de la vida consigue que no tengas al ver esta serie más problemas de los que ya tienes. Hay series que son dramas, que justo van más a los problemas o cosas impactantes. En Friends tratan que todo sea muy light. Por eso no reflejan ningún drama y lo importante es el humor. Entonces, yo no diría que la veía por evasión. Sobre todo a mí me gustaba para pasar un rato divertido viendo algo que era muy agradable.

(Entrevista en profundidad, 28 años, hombre, gerente)

Dentro de este escenario de lo agradable, el desarrollo de las historias representadas en esta serie es siempre positivo. Este tema de la visión esperanzadora y vitalista que desprende va a ser muy recurrente en el discurso de sus seguidores y potencia que quieran ver esta comedia de situación. Se tocará este tema más adelante pero se considera oportuno mencionar en este apartado dedicado al consumo cómo ver Friends es una actividad resfrescante y liberadora de problemas para su audiencia, algo que fomenta la capacidad de evasión que proporciona.

En definitiva, se pone de relieve cómo la necesidad de entretenimiento hace que la audiencia consuma un producto humorístico de formato ágil, consumo rápido y gratificación instantánea. Sobre todo, hay que recalcar cómo el consumo de esta serie ha estado generado por la atracción hacia un mundo de ficción cómodo de consumir y que se presenta agradable de ver. Esta atracción por este mundo ha hecho que parte de la audiencia tuviera incluso una cita diaria frente al televisor. Además, la facilidad y comodidad en el consumo ha hecho que muchos seguidores se hicieran con capítulos en DVDs, lo que ha llevado consigo la posibilidad de un consumo más intenso. 


\section{La actitud de los espectadores ante el universo “americano" representado}

\subsection{El nivel de implicación del fan}

Si se sigue la idea barajada por algunos autores sobre el nivel de implicación con una serie o involvement (Liebes, Katz, 1990), en este estudio se discriminó a los espectadores que más alta implicación había tenido con la serie para ver si existían variaciones significativas. Para ello, se cruzaron las tres variables que mejor podían graduar el nivel de implicación: seguimiento de la serie, grado de entretenimiento y nivel de gratificación a través del humor. Así que se tomaron como referencia las tres preguntas de la encuesta que hacían alusión directa a estas tres cuestiones. La primera se les pidió que definieran su grado de seguimiento de la serie y se les dio a elegir entre haber seguido prácticamente toda la serie, haber visto algunas temporadas pero no la serie completa o haber visto ocasionalmente la serie. En la segunda variable utilizada se les preguntó cómo consideraban la serie y se les dio a elegir entre tremendamente entretenida, muy entretenida, entretenida sin más, poco entretenida y nada entretenida. En último lugar, con la tercera variable utilizada se quiso evaluar cuánto de graciosa era Friends para su audiencia y pudieron elegir entre tremendamente graciosa, muy graciosa, algo graciosa, poco graciosa y nada graciosa.

Como ya se ha puesto de manifiesto que el público escogido como muestra en la encuesta eran principalmente fans caracterizados por su alto seguimiento y gratificación de la serie, se decidió separar a aquellos espectadores que daban los valores más altos en estas tres variables, es decir, que representaran una actitud extrema: haber seguido prácticamente toda la serie y considerarla tremendamente divertida y graciosa. En consecuencia, se hizo dos grupos: uno compuesto por este grupo de espectadores con muy alta implicación y otro con el resto de la muestra. Siguiendo esta clasificación, se crearon dos variables distintas que medían el grado de implicación de los seguidores: una variable con los que presentaban una muy alta implicación y otra con los que mostraban menor implicación. Se reveló que 1.385 fans estarían dentro del grupo de muy alta implicación, lo que conforma un 56,5\% de la muestra. El grupo de menos implicación representaría el 43,5\% de los encuestados con 1.065 espectadores. [Véase tabla 6]. La primera conclusión que se puede sacar de esta comparación es que son 
mayoría los altamente implicados con la serie frente a los que están menos implicados. Interesa ahora descubrir si existen diferencias reseñables entre ambos. Para ello, se cruzaron estos dos grupos con algunas preguntas que se formularon en la encuesta.

Tabla 6: Grado de implicación de los seguidores

\begin{tabular}{|c|c|c|}
\hline MUY ALTA IMPLICACIÓN & MENOS IMPLICACIÓN & TOTAL \\
\hline 1.385 seguidores & 1.065 seguidores & 1491 seguidores \\
\hline $56,5 \%$ & $43,5 \%$ & $100 \%$ \\
\hline
\end{tabular}

Si se cruza la variable que se ha creado para medir la implicación de la audiencia con la variable de género se observa que existe alguna variación significativa entre los participantes menos implicados con el producto en función del género. Esta relación apunta a que los hombres tienden a implicarse más con la serie si se compara con las mujeres. Los hombres que presentan una muy alta implicación con la serie superan a aquellos cuya implicación es más baja en 3,5 puntos porcentuales. Ocurre exactamente lo contrario en relación con la audiencia femenina. En este caso, son las mujeres con baja implicación las que superan en 3,5 puntos porcentuales a aquellas con muy alta implicación. Por lo tanto, sí que parece sintomático que dentro de los hombres sean mayoría los que presentan una implicación muy alta y que dentro de las mujeres sean mayoría aquellas que presentan una baja implicación con la serie. Parece que son los hombres quienes tienden a involucrase más con Friends. [Véase tabla 7].

Tabla 7: Grado de implicación por sexo

\begin{tabular}{|c|c|c|}
\hline SEXO/IMPLICACIÓN & $\begin{array}{c}\text { MUY ALTA } \\
\text { IMPLICACIÓN }\end{array}$ & BAJA IMPLICACIÓN \\
\hline Hombre & $62,4 \%$ & $58,9 \%$ \\
\hline Mujer & $37,6 \%$ & $41,1 \%$ \\
\hline Total & $100 \%$ & $100 \%$ \\
\hline
\end{tabular}




\subsection{Grado de implicación y tipo de historia. Entre lo universal y lo propio de} la ficción

Especialmente interesante es el cruce de grado de implicación por tipo de historia ya que refleja la relación que se establece entre los espectadores y el universo representado. La encuesta mostraba ya datos esclarecedores. Recordemos que al preguntar a los encuestados qué tipo de historias se contaban en Friends, el 63\% de la audiencia española encuestada declaró que las historias narradas en esta comedia de situación son universales, el 17,3\% consideraba las tramas propias de la ficción y solamente un 6,5\% llegó a afirmar que las historias representadas en Friends eran exclusivamente americanas. En líneas generales, la audiencia española conoce la ciudad de Nueva York perfectamente y cree que la serie incide muy bien en esa imagen de ciudad vertiginosa en la que es muy difícil profundizar en relaciones duraderas.

Los espectadores con muy alta implicación aventajan en 17,5 puntos porcentuales a los menos implicados en la pregunta que relacionaba Friends con una temática universal. Una tónica que se invierte respecto a los que consideran los temas representados en esta serie como propios de la ficción, ya que los seguidores menos implicados aventajan a los muy implicados en 13,6 puntos porcentuales. Esta asociación pondría de manifiesto un aspecto bastante interesante. Cuanta menos implicación hay con la serie en términos de consumo y gratificación, más distanciamiento hay con el mundo representado en la serie. Es decir, cuanta menos implicación hay con la serie más férrea es la barrera que separa la ficción con la realidad y los espectadores tienden a considerar ese universo propio como un mundo ajeno a ellos y a la vida cotidiana. Y al contrario, cuanto mayor sea la implicación con la serie en términos de consumo y gratificación, se tiende a calificar la serie con una temática universal [Véase tabla 8].

Tabla 8: Tipo de historia por grado de implicación

\begin{tabular}{|c|c|c|}
\hline $\begin{array}{c}\text { TIPO DE HISTORIA/ } \\
\text { IMPLICACIÓN }\end{array}$ & $\begin{array}{c}\text { MUY ALTA } \\
\text { IMPLICACIÓN }\end{array}$ & MENOS IMPLICACIÓN \\
\hline $\begin{array}{c}\text { Exclusivamente } \\
\text { americana }\end{array}$ & $5,4 \%$ & $7,9 \%$ \\
\hline Universal & $70,6 \%$ & $53,1 \%$ \\
\hline
\end{tabular}




\begin{tabular}{|c|c|c|}
\hline Propia de la ficción & $11,4 \%$ & $25 \%$ \\
\hline Otros & $12,5 \%$ & $13,9 \%$ \\
\hline
\end{tabular}

Completemos ahora estos datos porcentuales con información más cualitativa proveniente de los grupos de discusión y las encuestas en profundidad.

En primer lugar, los espectadores españoles muestran una gran familiaridad con el género de la comedia de situación y una proximidad con la realidad estadounidense representada en Friends. Sobre todo, presentan una gran cercanía hacia Nueva York, la ciudad en la que se desarrolla la acción, como afirma este espectador:

Todo el mundo conoce Nueva York. Esta ciudad es un 2 por ciento de la cultura norteamericana. Sin embargo, la gente lo ve como un 95 por ciento de lo que es Estados Unidos. Es la ciudad mejor conocida de todas. Todos tenemos una idea de qué es Nueva York y de cómo funcionan las cosas ahí. Ese carácter de la gente descolocada, de la gente que va conociendo unos a otros y sin acabar de profundizar en relaciones duraderas. Esa es la imagen que tenemos de Nueva York. De esa manera, la serie creo que incide en todo eso. Joey no sé con cuántas chicas puede salir, Rachel también está con cantidad de gente. Sin embargo ellos se configuran como un núcleo defendiéndose de toda esa vorágine de movimiento que hay en Nueva York. Creo que se presentan como personajes muy débiles que están soltados en la jungla que es Manhattan, la gran isla, y como ellos se protegen unos a otros. Del mismo modo que nosotros a los 20 años salimos de la universidad. Tenemos miedo, no sabemos dónde vamos a ir, si vamos a tener una casa, el trabajo. Tenemos todos nuestros miedos y sin embargo también buscamos nuestros núcleos de amigos para poder defendernos de todo eso que está ocurriendo a nuestro alrededor.

(Entrevista en profundidad, hombre, 26 años, arquitecto)

Este seguidor plantea una cuestión interesante ya que empieza su declaración afirmando que Nueva York es el centro de la cultura de Estados Unidos y, en seguida, hace una aplicación práctica para llevar esas historias a su vida personal. Hay que destacar, por tanto, la fuerza evocadora de ese universo recreado en torno a la ciudad de Nueva York y que consigue hacer esa realidad propia.

Por otro lado, la generación a la que se dirige la serie está muy acostumbrada a las series americanas y considera que la realidad americana forma parte ya de la sociedad española: 
A la generación a la que se puede dirigir más estamos tan sumamente acostumbrados a ver productos de Hollywood, a ver series americanas, y tal, que aunque igual no somos conscientes del todo, la realidad americana es parte de nuestra vida también, en el sentido de que, nos están haciendo vivirla. Entonces, bueno, pasa cuando vas por primera vez de viaje a Estados Unidos, dices: "Esto lo he visto ya. Aquí he estado". Pero, realmente nunca has estado, lo has visto. Y, bueno, al haber tenido tantos productos americanos aquí en la televisión y en el cine, pues bueno, hay una afinidad, por así decirlo, yo creo, y sí que ves las diferencias salvables que hay, pero, bueno, al estar tan acostumbrado. No es algo que digas, no es mi sociedad o lo que sea (sic).

(Entrevista en profundidad, 28 años, hombre, gerente).

Varias afirmaciones de este espectador son interesantes. En primer lugar, habla de un acostumbramiento, es decir, que ha visto tantas series y películas provenientes de Estados Unidos que la vida americana representada se le presenta como su propia sociedad. Este espectador declara que la ficción norteamericana, tanto series como películas, "les están haciendo vivir” la realidad americana. Esto pone de manifiesto que las películas y las series de televisión han sido, precisamente, quienes más han contribuido a esta cercanía de lo americana en España y, en segundo lugar, que esto se consigue dependiendo del grado de implicación o involvement con ese universo de ficción.

Respecto a los valores representados, la representación de la familia y el trabajo son consideradas por la audiencia española como típicos americanos. En relación con estos dos valores se establece una fuerte conexión entre lo representados y su propia vivencia como espectadores españoles. Respecto a la recepción de la familia, hay que señalar dos cuestiones. En primer lugar, los espectadores perciben que los personajes huyen constantemente de sus familias porque son poco normales. Solamente Ross y Mónica tienen unos padres aparentemente felices. El resto tiene unas familiar poco convencional:

Las familias de los protagonistas son bastante "chungas". Por ejemplo, el padre de Phoebe es un tío extrañísimo que aparece y desaparece de vez en cuando. Luego Chandler. Yo si tuviera un padre que se ha operado y canta en un club de Las Vegas, ipues no sé si iría muchas veces a cenar con él! O Joey, que tiene un montón de hermanas pero en el capítulo en el que salen dan mucho miedo. ¡Nadie quiere tener esas hermanas! Es que todos tienen familias muy raras. La exageración de esos padres tan raros te lleva a que también puedan exagerar el hecho de que no están nada con ellos. Su familia realmente no son sus padres. Yo creo que eso es lo que impulsa que la familia sean los otros cinco amigos.

(Grupo de discusión, 19 años, hombre, estudiante universitario) 
Por otro lado, una de las peculiaridades en la recepción de la representación de la familia entre el público español radica en su clara identificación con el prototipo de familia americana, muy alejada de la típica familia española:

Son típicos de la media americana. No de España porque aquí somos como más familiares. Más cercanos. Allí los jóvenes son muy independientes de sus padres. Los padres de Mónica y Ross que son los típicos americanos pero a ella su madre la ha maltratado por ser gorda. De cara a fuera como muy perfectos pero luego entre ellos no se podían ni ver. Y los padres de Rachel, pues muy perfectos pero entre ellos no se soportan y se separan. En fin, no lo veo tanto así aquí.

(Entrevista en profundidad, 26 años, hombre, gestor analista-financiero)

Por otro lado, interesa conocer cómo recibe la audiencia española las relaciones laborales en Friends, una serie protagonizada por unos jóvenes que han terminado sus estudios y emprenden un camino profesional. Lo primero que hay que matizar es que el trabajo no es un tema recurren en las tramas de la serie debido a las peculiaridades propias de la producción. Son pocas las escenas en las que se ven los lugares de trabajo de los protagonistas y aparecen casi siempre por alusión. Ante esto, a los espectadores españoles les sorprende mucho que los seis personajes apenas trabajen. Además, el mundo laboral es retratado como humillante. Lo más significativo es que el público español considera que la representación del trabajo en esta serie es propia de la "cultura del triunfador" estadounidense en la cual quien no triunfa en el trabajo es un perdedor:

El mundo laboral en Friends es el propio de la cultura americana: si no eres alguien en el trabajo, no eres nadie en la vida. Para ellos el trabajo tiene una importancia vital. Y siempre es competir, ascender y llegar a ser mejor. Yo creo que en España nos tomamos las cosas de otra manera.

(Grupo de discusión, 30 años, hombre, biólogo)

Respecto a la representación de otros valores como la amistad y el amor, la audiencia muestra actitudes diferentes que varían desde quienes la consideran real o ideal hasta quienes piensan que es más propia de la ficción. Sin embargo, es significativo cómo ningún espectador las relaciona con una representación propia de la cultura americana. 


\section{Conclusión:}

Esta investigación subraya la importancia del concepto de entretenimiento o disfrute (pleasure) para entender la experiencia de la audiencia cuando ve una serie como Friends. La mayoría de la literatura revisada ya apuntaba al pleasure como concepto clave en la experiencia televisual y este estudio reitera su relevancia como objeto de estudio. Se ha comprobado que el entretenimiento que plantea Friends está relacionado con determinados usos y gratificaciones de la audiencia. Además, las emociones que Friends es capaz de generar en sus espectadores hace que éstos tengan una particular actitud ante la realidad representada.

Este producto humorístico ha ofrecido todo un abanico de gratificaciones que van desde la puramente física -la relajación y liberación de tensión propia de la risahasta la anímica, propia de un producto que fomenta la evasión. Más allá del formato ágil y dinámico, los espectadores hablan de la atracción hacia un mundo que muestra la parte simpática y amable de la realidad.

Respecto a la recepción de los valores representados en Friends, se debe destacar la familiaridad con que la audiencia española se enfrenta tanto al género de las comedias de situación como a la ciudad americana donde se desarrolla la acción, Nueva York. Se observa, también, una identificación generacional entre los espectadores y los protagonistas de esta serie que les hace superar su distanciamiento geográfico.

La encuesta realizada entre los fans españoles de la serie revela cómo la audiencia no siente el universo de Friends como exclusivamente americano. Hay que subrayar la importancia de la variable de implicación con la serie o involvement- que mide el consumo y gratificación obtenida- ya que es la que explica la variación entre la audiencia que considera los valores representados en Friends como universales y quienes los considera propios de la ficción. Por lo tanto, se debe insistir en la universalidad de los temas tratados en esta serie.

A pesar de la acusada familiaridad y acostumbramiento hacia universos de ficción norteamericanos, se observa una actitud crítica hacia determinados valores representados como son la familia y el trabajo. La audiencia española cree que la representación de estos valores en Friends es propia de la cultura americana, algo que 
pone de manifiesto cómo los espectadores interpretan la realidad representada en esta serie desde el prisma particular de la cultura española.

\section{Referencias:}

ANG, I. (1985): Watching Dallas. Soap Opera and the Melodramatic Imagination, Routledge, London.

BAYM, N (2000): Tune In, Long On. Soaps, Fandom and Online Communities, Sage, Thousand Oak.

BILTEREYST, D. (1991): "Resisting American Hegemony: A comparative Analysis of the Reception of Domestic and US Fiction” en European Journal of Commmunication (6), pp. 469497.

BROOK, V. (2004), "Myth or Consequences: Ideological fault lines in The Simpsons" en ALBERTI, J. (ed.), Leaving Springfield. The Simpsons and the Possibility of Oppositional Culture, Wayne State University Press, Detroit.

CHITNIS, K. (2006): “(Dis)similar readings. Indian and American Audiences’ Interpretation of Friends” en International Communication Gazette 68 (2), pp. 131-145.

DHOEST, A. (2007), "Identifying with the nation: viewer memories of Flemish TV fiction" en European Journal of Cultural Studies, 10(1), pp. 55-73.

EHRENREICH, B.; HESS, E.; JACOBS, G. (2003), "Beatlemania. Girls Just Want to Have Fun” en BROOKER, W.; DEBORAH, J. (eds.), The Audience Studies Reader, Routledge, London, pp. 180-184.

FIGUERAS, M. (2006): “Las series son como la vida”. El significado para las adolescentes de la ficción televisiva. Disponible http://www.educacionenvalores.org/article.php3?id_article=680. Fecha de consulta: 28 de junio de 2007.

GRANDÍO, M. (2007): "Reflexiones sobre los estudios de las audiencias en España. Carencias y retos de futuro” en Sphera Publica, Número Especial, pp. 157-172.

HARRINGTON L.; BIELBY D. (2005): "Global Television Distribution: Implications of TV 'Traveling' for Viewers, Fans, and Texts” en American Behavioral Scientist 48 (7), pp. 902920.

HUERTAS, A.; FRANÇA, M. E. (2001): "Una aproximación a cómo contribuye la televisión en la construcción del yo" en Zer. Revista de estudios de comunicación, 11. Disponible en: http://www.ehu.es/zer/zer11web/huertas.htm. Fecha de consulta: 28 de junio de 2007.

JENKINGS, H. (1992), Textual Poachers: Television Fans and Participatory, London, Routledge. 
JENKINS, H. (1995), "Do You Enjoy Making the Rest of Us Stupid?: alt.tv.twinpeaks, the Trickster Author, and the Viewer Mastery" en LAVERY D., (ed.): Full of Secrets. Critical Approaches to Twin Peaks. Detroit, pp. 51-69.

KUIPERS, G. (2006): "Television and Taste Hierarchy: the Case of the Dutch Television Comedy” en Media, Culture and Society, 28(3), pp. 359-378.

LACEY, J. (2002), "One for the Boys? The Sopranos and its Male British Audience” en LAVERY, David (ed.), This Thing of Ours: Investigation The Sopranos, Columbia University Press, New York, pp. 95-105

LIEBES, T.; KATZ, E. (1990), The Export of Meaning. Cross-cultural Readings of Dallas, Oxford University Press, New York.

MONTERO, Y. (2006): Televisión, valores y adolescencia. Barcelona, Gedisa.

OLSON, S. (1999), Hollywood Planet: Global Media and the Competitive Advantage of Narrative Transparency, Mahwah, Lawrence Erlbaum.

PINDADO, J. (2006), "Los medios de comunicación y la construcción de la identidad adolescente” en Zer 21. Pp.11-22.

RADWAY, J. A. (1991): Reading the Romance. Women, Patriarchy, and Popular Literature: University of North Carolina Press, Chapel Hill. Primera edición en 1984.

SCHRØDER, K. (1988), "The Pleasure of Dinasty. The Weekly Reconstruction of SelfConfidence” en DRUMMOND, P.; PATERSON, R., Television and its audience, British Film Institute, London, pp. 61-82.

SPENCE, L. (2005): Watching Soap Operas. The Power of Pleasure, Wesleyan University Press, Middletown. 